\title{
Message From the Editors to Our Reviewers
}

José G. Merino, MD, MPhil, Olga Ciccarelli, MD, PhD, FRCP, Bradford B. Worrall, MD, MSc, Anthony A. Amato, MD, Rebecca Burch, MD, John R. Corboy, MD, Josep O. Dalmau, MD, PhD, Jonathan Graff-Radford, MD, Jennifer Graves, MD, PhD, MAS, Peter Hedera, MD, PhD, Linda A. Hershey, MD, PhD, Barbara C. Jobst, MD, PhD, Stefan M. Pulst, MD, Reneé A. Shellhaas, MD, MS, and Roy E. Strowd, III., MD

Neurology ${ }^{\circledR}$ 2021;96:1-9. doi:10.1212/WNL.0000000000011204

Neurology ${ }^{\oplus}$ manuscript submissions continue to increase every year; mid-2020 saw a large influx of manuscripts related to complications of coronavirus disease 2019 (COVID-19), many of which went through an expedited peer-review process. The journal received 4301 new and 691 revised manuscripts between April 1, 2020, and September 30, 2020 (compared to 3336 new and 597 revised manuscripts during the same period in 2019). We received 7158 peer reviews (compared to 4455 during the same period in 2019) evaluating these papers.

Only a minority of submitted manuscripts can be published. Decisions regarding which articles will most benefit readers and improve patient care are often difficult. Your comments regarding uniqueness of study populations, novel methods, studies that are especially educational, or new strategies for diagnosing and treating neurologic disease are immensely helpful. We are grateful for your cooperation in returning reviews within a timely manner despite your many other obligations. The benefit to authors and the journal from your feedback is extremely valuable; peer review is an essential mechanism for assessing quality of research and conferring credibility to the content we publish.

We also appreciate the constructive feedback by reviewers of manuscripts submitted to the Resident \& Fellow Section of Neurology. Your comments are highly constructive to junior authors striving to improve their clinical and scientific writing abilities.

Please follow the guidelines for reviewing papers accessed by selecting the Information for Reviewers (IFR) link at n.neurology.org/submit/submitreview. The IFR provides information on expectations of reviewers regarding confidentiality, timeliness, and reviewer conflicts of interest. It also provides instructions for formatting the comments to editors and authors to enable the most effective communication with authors.

Please email journal@neurology.org if you would like to do more reviews or if you have never reviewed for the journal but are interested in doing so. Include a description of your credentials and expertise in the areas in which you are qualified to review.

The reviewers listed below with 1 asterisk have reviewed 5 or more papers between April 1, 2020, and September 30, 2020. Two asterisks indicate that the reviewer has reviewed 10 or more manuscripts during the same period. This list includes those reviewers who returned a review or reviews of initial submissions (re-reviews of the same manuscript are not included).

Whitley W. Aamodt*

Ashley Aaroe

Jan O. Aasly

Hesham Abboud

Ahmed Abdelhak

Mohammad Ammar

Abdulrazzak

Nicholas S. Abend
Erin L. Abner*

Lauren E. Abrey

Martina Absinta

Mohammad Abu-Rub

Lealani Mae Acosta**

Gyula Acsadi

David D. Adams

Harold P. Adams*
Heather Adams

Jamie L. Adams

Amelia Adcock

Malik Adil ${ }^{* *}$

Mohit Agarwal

Nitin Agarwal

Puja Agarwal

Shashank Agarwal**
Federica Agosta**

Samrah Ahmed

Rebecca Ahrens-

Nicklas

Jessica University

Ailani

Laura M. Airas

Gülden Akdal
Rizwan S. Akhtar

Nazem Akoum

Haitham Al Ashry

Thomas A. Ala

Steven M. Albert

Roger L. Albin

Megan Christine

Alcauskas* 
Yazan J. Alderazi

Andrei V. Alexandrov

Alison Alford

Alicia Algeciras-

Schimnich

Farwa Ali*

Yasir Al-Khalili

Jeffrey A. Allen*

Leonardo Almeida

Michael L. Alosco

Saud Alsahli*

Ola A. Alsalman

Rustam Al-Shahi

Salman

Jumana T. Alshaikh*

Claudia Altamura

Robert Altman

Jordan Yardain Amar*

Rebecca Amariglio

Maria Pia Amato

Sebastián F. Ameriso

Beau M. Ances

Christopher D. Anderson

Craig Anderson

David C. Anderson

X. Michelle

Androulakis

Corrado I. Angelini*

Sabrina Anticoli

Angelo Antonini

Yaacov Anziska

Hugo J. Aparicio

Liana G. Apostolova

Stephen Aradi

Antonio Arauz

Francesco Arba*

Kristy Arbogast

Juan F. Arenillas

Charles E. Argoff

Zohar Argov

Hiba Arif Haider

Hisatomi Arima

Carmel Armon**

Floyd Daniel

Armstrong

Melissa J. Armstrong*

Georgina Arrambide

Filippo Arrigoni

Ethem Murat Arsava

Kapil Arya*

Ravindra Arya

Masato Asahina

Eishi Asano

Alberto Ascherio

Sharon Ash

Giovanni Assenza
Masharip Atadzhanov

Nazem Atassi

Bertrand Audoin

Sanford Auerbach

Eitan Auriel

Robert Avery

Cenk Ayata

Scott Ayton

Ahmad Aziz

Tipu Z. Aziz

Suzanne Babyar

Neeraj Badjatia

Johan-Emil Bager

Sergio Bagnato

James F. Bale, Jr.

Robert W. Baloh

Oliver Bandmann

Uddyalok Banerjee

Anna M. Bank

Sergio Baranzini

Richard L. Barbano

Frederik Barkhof*

Lisa L. Barnes

Carolina Barnett

Jean-Claude Baron

William B. Barr

Luca Bartolini

Brandon R. Barton*

Russell E. Bartt

Joshua I. Barzilay

Rifaat M. Bashir

Claudio L. Bassetti

James R. Bateman

Stephanie Baulac

Margaret L. Bauman

Christian R. Baumann

Tobias Bäumer

Sallie A. Baxendale

Carl W. Bazil

David Bearden

Lori Beason-Held

Martina Bebin

Werner J. Becker

Peter Bede

Edward M.

Bednarczyk

Ettore Beghi

Alexandre Bejanin

Samir R. Belagaje

Olivia Belbin

Vincenzo Belcastro

Álvaro Beltrán

Corbellini

David R. Benavides

Selim R. Benbadis

Barbara Brigitta

Bendlin
Giulia Benedetti

Sandor Beniczky

Sarah M. Benish

Andreana Benitez

Julián Benito-Leon

Elinor Ben-Menachem

David A. Bennett

Wyatt P. Bensken

Richard T. Benson

Roy G. Beran

Shannon Beres

Anne T. Berg**

Joseph R. Berger

Klaus Berger

Samuel F. Berkovic

Aaron L. Berkowitz

Madison M. Berl

Robert A. Bermel

Andrea Bernasconi*

Neda Bernasconi*

James L. Bernat

Julie Bernhardt

Charles Bernick

Anna Bersano

Jimmy Berthaud

Edward H. Bertram

Anand Bery*

Frank Besag

Lauren A. Beslow

Sonam Bhalla

Poonam Bhatia

Shamik Bhattacharyya

Italo Biaggioni

Marino Muxfeldt

Bianchin

Razieh Bidhendi

Yarandi

Geert Jan Biessels

Alessandro Biffi*

Murat Bilgel

Jose Biller

Michel M. Billiard

Devin K. Binder

Malcolm Binns

Gretchen Lano

Birbeck

Antje Bischof

Gerard N. Bischof

Alexander R. Bisdorff*

Francesca Bisulli

Andrew Fabian Bleasel

Ingmar Bluemcke

Helena Blumen

Sergiu C. Blumen

Amelia K. Boehme

Christopher J. Boes

Sylvia M. Boesch
Julien Bogousslavsky

Richard W. Bohannon

Nicolaas I. Bohnen

Aldo Bonaventura

Paul Boon

Alexis Boro

Soo Borson

Hugo Botha*

Dennis Bourdette**

Rose-Mary N.

Boustany

James H. Bower

Alex Bowers

Laura S. Boylan

Serge Brand

Ethan S. Brandler

John Frederick

Brandsema*

Robynne Braun

John C. Breitner

Matthias Brendel

David A. Brenner

J. Nicholas Brenton

Farren Basil Shaw

Briggs

Vera Bril*

Benjamin $\mathrm{H}$.

Brinkmann

Jeffrey W. Britton

Tom Britton

Bruno Brochet

Kathrin Brockmann

Joseph P. Broderick

Michael C. Brodsky

Amy Brodtmann**

Mark B. Bromberg

Benjamin Rix Brooks

David James Brooks

Matthijs C. Brouwer

Devin L. Brown**

Wallace Brownlee*

Oliviero Bruni

Iris Brunner

John C.M. Brust**

Maria Jose Bruzzone

Giraldez

Rachel F. Buckley**

Marion S. Buckwalter

Andrew E. Budson

Catherine Buettner

Krzysztof Bujarski

Zachary Bulwa

Emanuele Buratti

James F. Burke*

David Bruce

Burkholder

Jorge G. Burneo
Joseph D. Burns*

Joshua Burns

Richard Burt

Ittai Bushlin

Saif Bushnaq

Cheryl D. Bushnell

Neil A. Busis*

Nitin Butala

John A. Butman

Russell J. Butterfield

Helmut Butzkueven

Katsiaryna Bykov

Richard Byrne

Dominique A. Cadilhac

Paolo Calabresi

Peter A. Calabresi

Daniel Calame

Fiona J. Caldwell

Brian C. Callaghan*

Peter R. Camfield

Richard M. Camicioli

Millene Camilo

Bruce C.V.

Campbell**

Meghan C. Campbell

Philippe Campeau

Stephen C. Cannon

Louis R. Caplan

Stefano F. Cappa

Roberto H. Caraballo

Martha Carlson

Michelle C. Carlson

S. Thomas Carmichael

Antonio Carolei

Arturo Carpio

Aisling S. Carr

David B. Carr

Jonathan Carr

Emmanuel Carrera

Joseph Carrera

Nuria Carrillo

Ian Carroll

Michael S. Cartwright*

Gabriela F. Carvalho

Gregory D. Cascino

Richard J. Caselli

Ilaria Casetta

Luis Castilla-Guerra

Pablo R. Castillo

Sergio A. Castillo-

Torres

Paola Cavalla

Andrea Eugenio

Cavanna

John N. Caviness

Fernando Cendes* 
Bruno Censori

Mara Cercignani

Anna M. Cervantes-

Arslanian

Sabina Cevoli

Colin Chalk

Micaela Y. Chan

Richard Chan

Cherylee W.J. Chang

Jason J. Chang

Michael J. Chao

Silvia Chapman

Declan T. Chard

David Charles

Seemant Chaturvedi**

Vinay Chaudhry

Gerardo Chavira

Hernandez

Alex Bou Chebl

Celia S. Chen

Ching-Jen Chen

Chiung Chu Chen

Honglei Chen

John Jing-Wei Chen

Kewei Chen

Patrick Chen*

Robert Chen

Timothy Chen

Wei Chen

Wei-Liang Chen

Yuying Chen

Neena Cherayil

Luisa Chiapparini

Marc I. Chimowitz

Jerome H. Chin

Adriano Chio*

Joon Yul Choi

Catherine D. Chong

Michael R. Chong

Raymond Chong

Kelvin L. Chou*

Miguel Chuquilin

Bart Chwalisz

Antonio Ciacciarelli

David X. Cifu

Carla Cirillo

Daniel O. Claassen*

Kristl G. Claeys

Joseph Classen

Benjamin R. Claytor**

David B. Clifford

Derin Cobia

Keith A. Coffman

Hugo Cogo-Moreira

Bruce H. Cohen

Jeffrey A. Cohen

Fleur Cohen Aubart
John W. Cole

James G. Colebatch*

Alasdair Coles

Devon S. Conway

Elizabeth A. Coon

Charlotte Cordonnier

David R. Cornblath

Denise D. Correa

Nick Corriveau-

Lecavalier

Pietro Cortelli

Irene Cortese

Stephanie A.

Cosentino

Daniel Costello

Fiona E. Costello

James R. Couch, Jr.

Bruce M. Coull

Timothy J. Counihan*

Jonathan M. Coutinho

Bruce Anthony

Campbell Cree

Amy Crepeau

Claire J. Creutzfeldt*

Susan R. Criswell

Nathan E. Crone

Carolyn Cronin

Salvador Cruz-Flores

Collin J. Culbertson

Antonio Culebras*

Jeremy K. Cutsforth-

Gregory** $^{* *}$

Gary R. Cutter

Aman Dabir*

Marinos C. Dalakas

Russell C. Dale

Caroline Dallaire-

Théroux

Josep O. Dalmau

Eleonora Silvana

D'Ambrosio

Kristen Dams-

O'Connor

Louis T. Dang

Ryan Darby

Felice D'Arco

Lincoln S. Darla

Basil T. Darras*

Jean-Francois

Dartigues

Sirwan K.L. Darweesh

Rohit Das*

Neil Datta

Kathryn A. Davis

Stephen M. Davis

Thomas L. Davis

Gregory Scott Day*
Rob M.A. de Bie

Mamede de Carvalho*

Gabriel R. de Freitas*

Adam de Havenon

Suzanne M. de la

Monte

Frank-Erik de Leeuw*

Andres De Leon-

Benedetti*

Gian Marco De

Marchis

Jerome de Seze

Roberto De Simone

Leonardo Cruz de

Souza

Marianne de Visser

Linda de Vries

Lisa M. DeAngelis

Aman Deep*

Giovanni Defazio

Diana Degan

Ellen M. Deibert

Teodoro Del Ser

Guillermo Delgado-

$$
\text { García** }^{*}
$$

Bart M. Demaerschalk

Scott T. Demarest

Naiara Demnitz

Tom den Heijer

Christian Denier

Martin Dennis

Mary Carter Denny

E. Michelle DePoy

Colin P. Derdeyn

Tobias J. Derfuss

Masoom Desai

Maxime Descoteaux

Michel Desmurget

Mariel Brooke

Deutsch

Gabrielle DeVeber

Orrin Devinsky

Marianne DeVisser

Anita Devlin

Feza Deymeer*

Klodian Dhana

Nyssa d'Hedouville

Davide Tiziano Di Carlo

Francesco Di

Lorenzo*

Daniel Garbin Di Luca $^{* *}$

Emilio Di Maria

Ramon Diaz-Arrastia

Martin Dichgans

Nico Jean Diederich
Jose Danilo Bengzon

Diestro

Marianne Dieterich

Jacopo C. DiFrancesco

Kathleen B. Digre

Rick M. Dijkhuizen

Dale Ding

Djurdja Djordjevic

Dennis J. Dlugos

Bruce H. Dobkin

Ruth Dobson*

James D. Doecke

Okan Dogu

Magdalena E.

Domellöf

Rogelio Dominguez

Moreno*

Lisanne

Dommershuijsen

Junling Dong

Richard L. Doty

Fergus Doubal

Christopher Doughty

Vanja C. Douglas

James J. Dowling

Daniel B. Drachman

Bogdan Draganski

Jichen Du

Rose Du

Ranjan Duara

Francois Dubeau

Divyanshu Dubey

Janet Dubinsky

Richard M. Dubinsky*

François Ducray

Marco Duering

Kevin Duff

Joseph R. Duffy

Jessica Duis

Sean P. Dukelow

Mario F. Dulay

John S. Duncan

Thierry P. Duprez

Luc Dupuis

Pierre Duquette

Barbara A. Dworetzky

P. James B. Dyck

Tomasz Dziedzic

K.P. Ebmeier

Lorraine L. Edwards

Mark J. Edwards

Eric R. Eggenberger

Juergen Eggers

Rodger J. Elble

Merrill F. Elias

Mitchell S.V. Elkind

Jeremy A. Elman
Ronald Emerson

Jerome Engel, Jr.

Stephanie Enner

Erik R. Ensrud

Leon Epstein

Sofia H. Eriksson

Deniz Erten-Lyons

Arman Eshaghi**

Alberto J. Espay

Mill Etienne

Mahyar Etminan

Nima Etminan

Denis A. Evans

Stefan Evers

Joshua B. Ewen

Michael Ewers

Camilo E. Fadul

Guido J. Falcone

Jose Marcelo Farfel

Nathan Farkas*

Alfonso Fasano*

Klaus Fassbender

Paolo Federico

Dominic B. Fee*

Michael G. Fehlings

Seyed-Mohammad

Fereshtehnejad

Luigi Ferini-Strambi

Tanis J. Ferman

Juan Diego Fernandez

Daniel Ferreira

A. James Fessler

Anteneh M. Feyissa

Erin Rebecca Fiedler

Terry D. Fife

Massimo Filippi*

Alessandro Filla

Christopher M. Filley

Carsten Finke

Robert S. Fisher

Edward F. Fitzgerald

Eoin P. Flanagan

Jeff Fletcher**

Alexander C. Flint

Charles C. Flippen II

Enrico Flossmann

Ana Catarina Fonseca

Rafael Fonseca

Gary A. Ford

Laura A. Foster

Alexandra Foubert-

Samier

Christina N. Fournier

Robert J. Fox ${ }^{* *}$

Susan Fox

Elizabeth Fracica

Silvana Franceschetti 
Samuel A. Frank

Gary M. Franklin*

Clare L. Fraser

John Alexander Fraser

Pietro Fratta

Daniel A. Freedman

Jacqueline French

Vera Fridman

Robert P. Friedland

Daniel Friedman

Deborah I. Friedman

Jennifer Friedman

Joseph H. Friedman*

Joseph Irwin Friedman

Richard Eugene Frye

Katherine A. Fu*

Jennifer E. Fugate

Shinsuke Fujioka*

Hiroshige Fujishiro

Hidenao Fukuyama*

Timothy Fullam

Victor S.C. Fung

Julio C. Furlan

Erin Furr Stimming

Ansgar J. Furst*

Julia Furtner

Lucia Fusco

Satish Gaddam

Douglas Galasko

Steven Galetta**

Cecile Gallea

Javier Andrés

Galnares-Olalde*

Charlene E. Gamaldo

Antonio Gambardella

Rachana Gandhi

Carlo Gandolfo

Florin Gandor

Vijay Ganesh

Gautam Ganguly

Xiang Gao

Paul A. Garcia

Rocio Carolina Garcia-

Santibanez

Andrew Gardner

Christopher Gardner

Ravindra Kumar Garg

Valentina Garibotto

Emilio R. Garrido

Sanabria

David S. Geldmacher

Marios K. Georgakis

Benjamin P. George

Ilena George**

Elizabeth E. Gerard

Christian Gericke

Menno R. Germans
Jeroen J.G. Geurts*

Milena Gianfrancesco

Christopher $\mathrm{H}$.

Gibbons

Nicola Giffin

Donald L. Gilbert

Gordon J. Gilbert

Dipender Gill

Frank Gilliam

Emily Gilmore

Wendy Gilmore

Antonino Giordano

Juan Domingo Gispert

Ezequiel Gleichgerrcht

David Gloss*

M. Maria Glymour*

Simon Glynn

Peter J. Goadsby

Hans H. Goebel

Sanjay Gokhale

Sankalp Gokhale

Stefan Martin

Golaszewski*

Lawrence I. Golbe

Daniel Gold

Ralf Gold

Stefan M. Gold

Daniel M. Goldenholz

Alica M. Goldman*

Serge Goldman*

Larry B. Goldstein**

Meredith R. Golomb

Camilo R. Gomez

David Gomez Andres

Julie Gonneaud

Nicole R. Gonzales**

Marco A. Gonzalez

Castellon*

Luis Nicolas Gonzalez

Castro*

Esteban Gonzalez-

Lopez

Paloma Gonzalez-

Perez

Clifton L. Gooch*

Howard Parker

Goodkin

James M. Gordon

Philip B. Gorelick

An Goris

Jean Gotman

Rebecca F.

Gottesman*

Rachel Gottlieb-Smith

Christopher

Gottschalk

Raghav Govindarajan
Namita A. Goyal

Hans J. Grabe

Jerome J. Graber**

William D. Graf

Neill R. Graff-Radford

Mark A. Granner

Cristina Granziera

Maria V. Grau-

Sepulveda

Alexander L. Green

Kemar E. Green

Steven M. Greenberg*

Trisha Greenhalgh

Beverly Greenspan

Robert S. Greenwood

Simon G. Gregory

Stefan Greisenegger

Robert C. Griggs

Joshua D. Grill

Timo Grimmer

Zachary M. Grinspan

Wolfgang Grisold*

Samuel Groeschel

Gary Gronseth**

Bradley A. Gross*

Catharina C. Gross

Murray Grossman

Scott N. Grossman

Michel Grothe

Michael Gruenthal

Quinn Grundy

Andrzej Grzybowski

Aashrai Sai Venkat

Gudlavalleti

Renzo Guerrini*

Amanda C. Guidon*

Kristin Guilliams

Deepak Gulati

Jeffrey L. Gunter

Harsh Vardhan Gupta

Himanshu Gupta

Mahmut E. Gurol

Deborah Gustafson

Jose Gutierrez

David H. Gutmann

Ludwig Gutmann**

Amy Katherine Guzik

Mary N. Haan

Yael Hacohen*

Ellen Merete Hagen

Thaddeus J. Haight

Timothy C. Hain

Daniel Hajioff

Rashmi Halker

Charles Byron Hall

Gabor Michael

Halmagyi*
John J. Halperin*

Michael Halstead

Ali G. Hamedani*

Roy H. Hamilton**

Harald Hampel

Zulfi Haneef

Oskar Hansson

Ihtsham U. Haq

Orla Hardiman

Noam Y. Harel*

Matthew Harms

Judith U. Harrer*

Madeleine Harrison

Adam L. Hartman**

Andreas Hartmann

Danielle Harvey

Anhar Hassan

Jason Hassenstab

Jaime Hatcher-Martin

Robert A. Hauser

W. Allen Hauser*

Maximiliano A.

Hawkes

Eleanor Hayes-Larson

Michael K. Hehir

Emerald G. Heiland

Kenneth M. Heilman

Jennifer Heim

Kristen Heinan

Ingo Helbig

Kerstin Hellwig

Christoph Helmchen

Catherine Helmer

Bernhard Hemmer

J. Claude Hemphill

Galen V. Henderson

Nils Henninger

John W. Henson

JoonNyung Heo

Karl Herholz

Bruce Hermann

David N. Herrmann

Andrew D. Hershey

Shawn Hervey-Jumper

Roger R. Hesselbrock

Amy B. Hessler

Geoffrey L. Heyer*

Daniel B. Hier

Emily Hill

Argye Elizabeth Hillis*

Jason Hinman

H.E. Hinson*

Kwo Wei David Ho*

Jeremy C. Hobart

Lisa D. Hobson-Webb

Sara E. Hocker

Eric P. Hoffman
R. Edward Hogan

Birgit Högl

Gunter U. Hoglinger

Anna DePold Hohler

Reinhard Hohlfeld*

Timothy J. Hohman

Samantha K. Holden

Victoria Holiday

Melissa F. Holmes

Trygve Holmoy

Hans Holthausen

Martin Holtkamp

Sue Hong

Babak Hooshmand*

Jennifer L. Hopp

Rita Horvath

George Howard

Virginia J. Howard

Sung-Tsang Hsieh

Le H. Hua

Jason H. Huang

Xuemei Huang

Yue Huang

Hanneke E. Hulst

Rachael Maree Hunter

Robert Hurford**

Aatif M. Husain

Masud Husain

Shaun A. Hussain

David Y. Hwang

Susan T. Iannaccone

Agustin Ibanez

Rebecca N. Ichord*

Masafumi Ihara

Takahiro Iizuka

Manabu Ikeda

Winfried Ilg

Manabu Inoue

Carolina Ionete

Sarosh R. Irani

David John Irwin*

Stefan Isenmann

Fabio M. Iwamoto*

Ifeanyi O. Iwuchukwu

Juha E. Jääskeläinen

Michael Jacewicz

Carlayne Jackson

Giris Jacob

Bradley S. Jacobs

Daniel H. Jacobs

Amanda L. Jagolino-

Cole

William J. Jagust

Assia Jaillard

Zeenat Jaisani

Michael L. James

Todd Janus 


Suman Jayadev
Jiann-Shing Jeng
Thomas M. Jenkins*
Christina Jern
Nathalie Jette
Jia Jianping
Glen Jickling**
Nicholas E. Johnson
David T. Jones
Lyell K. Jones*
Joost Louis Jongen
Justin T. Jordan
Keith Anthony
Josephs
Charuta N. Joshi
Sucheta Joshi
Raed A. Joundi
Anne Joutel
Juho Joutsa
Eric Jouvent

Ralph F. Jozefowicz

Vern C. Juel

Csaba Juhasz*

Larry Junck*

Jinsei Jung

Stephen P. Juraschek

Arash Kahrom

Eric A. Kaiser

Tomas Kalincik

Stiliyan N. Kalitzin

Bernadette Kalman

Lalit Kalra*

Sanjay Kalra

Hooman Kamel

Henry J. Kaminski*

Joseph Kamtchum

Tatuene

Peter B. Kang*

Ronald M. Kanner

Petr Kanovsky

Gregory Kapinos*

Peter W. Kaplan

L. Jaap Kappelle

Moira K. Kapral

Jaideep Kapur

Kush Kapur

Amel Karaa

Ioannis Karakis*

Chafic Karam*

Axel Karenberg

Kazuomi Kario

Matthias Karst

Dimitrios Karussis

Diego Kaski

Gregor Kasprian

Tareq Kass-Hout

Jan Kassubek
Mira Katan*

Mitsuhiro Kato

Aristeidis H. Katsanos

Zaza Katsarava

Jorge C. Kattah**

Hans D. Katzberg

Daniel I. Kaufer

David I. Kaufman

Keisuke Kawata

Mark Robert Keezer

Sarah Aminoff Kelley

Christoph Kellinghaus

Adam Kelly

Kimbra Kenney

David Kent

Kevin A. Kerber

Silke Kern

Wesley T. Kerr

Navin Kesari*

Tigran Kesayan*

Zafer Keser*

Sudha Kilaru Kessler

Imad Khan

Omar I. Khan

Pouya Khankhanian**

Ammar Kheder

Jessica Kiarashi

Philipp Kickingereder

Charles J. Kidd

Desmond Patrick Kidd

Kelley Kidwell

Karl D. Kieburtz

Stefan Kiechl

Matthew C. Kiernan

Joep Killestein*

Ronald J. Killiany

Beom Joon Kim

David Dongkyung

Kim

Ho Sung Kim

Ji-Soo Kim

Scott Y.H. Kim

Yosuke Kimura

A.J. Kind

Michael O. Kinney

Laurence J. Kinsella

Howard S. Kirshner*

Steven J. Kittner

Brad C. Klein

Christopher J. Klein*

Joshua P. Klein

Matthias Klein

Britany Klenofsky

Thomas Klockgether

Benzi M. Kluger

Karen J. Kluin

William E. Klunk
David S. Knopman**

Kelly Knupp

Erik J. Kobylarz

Marcus Werner Koch

Sebastian Koch

Lisa M. Koehl

Peter J. Koehler

Matthew A. Koenig

Hans-Christian

Koennecke

Susan Koh

Wolfgang Köhler

Brad Kolls*

Zdravko Kolundžić

Edina Komlodi-

Pasztor

Katie Kompoliti

Barbara S. Koppel

Igor J. Koralnik

Gideon Koren

Miikka Korja*

Vladimir S. Kostic

Prakash Kotagal

Vikas Kotagal

Sanjeev V. Kothare

Karim Hussein

Kotkata*

Prateeka Koul

Alain K. Koyama

Mariel Kozberg

Ingeborg Krägeloh-

Mann

Christopher Lawrence Kramer

Gregory L. Krauss

Michal Krawczyk

Magdalena Krbot

Skoric

Hubertus Kremer

Michael C. Kruer*

Lauren B. Krupp

Kristen M. Krysko*

Jens Kuhle

Walter A. Kukull

Jaime Kulisevsky*

Lewis H. Kuller

Hrishikesh Kumar

Ishani Kumar

Neeraj Kumar

Pradeep Kumar

Priya Kumthekar

Doris Kung

Nancy Kuntz

Ajay Kurani

Roger M. Kurlan

Tobias Kurth

Deena Kuruvilla
Renaud La Joie

David M. Labiner

Brittany Bolduc

Lachance

Daniel T. Lackland

David Lacomis

Catherine C. Lacroix

Kathrin LaFaver

W. Curt LaFrance Jr.

Eugene C. Lai

Alice Lam

Keng Lam*

Susan Landau*

Doriana Landi

Eric C. Landsness

Douglas R. Langbehn

Carl D. Langefeld

Annette M. Langer-

Gould

Dianne Langford

Maarten G. Lansberg

Giuseppe Lanza

Giuseppe Lanzino

Hans Lassmann

Julius Gene Silva

Latorre*

Larry Latour

Simona Lattanzi

Robert Laureno

Giuseppe Lauria

Eric C. Lawson

Rachael A. Lawson

Robert B. Layzer

Ronald M. Lazar

Stephanie L. Leal

Victoria M. Leavitt

Jérôme R. Lechien

Mark S. LeDoux**

Andrew Lee

Jong Lee

Keon-Joo Lee

Beth Leeman-

Markowski

Paul Leeson

Lisa R. Leftert

Alan D. Legatt

Stephane Legriel*

Dana Leifer

Peter Nigel Leigh

Richard Leigh

Enrique C. Leira

Regan Jo Lemley

Abhishek Lenka**

Ilo E. Leppik

Thabele M. Leslie-

Mazwi

Doris G. Leung
Sue E. Leurgans

Kerry H. Levin

Mindy F. Levin

Netta Levin

Steven R. Levine

Michael Levy

Ariane Lewis

Richard A. Lewis

Sara A. Lewis

Simon Lewis

Peter A. LeWitt

Cristian E. Leyton

Hong $\mathrm{Li}$

Linxin $\mathrm{Li}$

Shanshan Li

Weizhe Li

Yi Li

John W. Liang

Roland Liblau

Daniel J. Licht

David G. Lichter

Karen Lidzba

Teerin Liewluck

Maria Liguori

Fabricio O. Lima

Jia Lin*

Michelle P. Lin ${ }^{* *}$

Anita Lindmark

Vasileios-Arsenios

Lioutas

Richard B. Lipton*

Grant T. Liu

Renyu Liu

Xinfeng Liu

Yin Allison Liu**

John Livingston

Alberto Lleo*

Rafael Llinas

Thomas E. Lloyd

Sara Llufriu

Warren D. Lo

Eric L. Logigian

Giancarlo Logroscino*

Zachary London*

Erin Longbrake

Oscar L. Lopez

Aaron Sylvan Lord

Svetlana Lorenzano*

Phillip A. Low

Val J. Lowe

Janping Lu

Jian-Qiang Lu

Lin Lu

Christian Lucas

Andreas Luft

Forshing Lui

Dorothée E. Lulé 
Codrin Lungu

Dana Lustbader

Andrew L. Lux

Jan Nils Lycke

Patrick D. Lyden

Michael J. Lyerly

Sharon G. Lynch

Ariel M. Lyons-

Warren ${ }^{* *}$

Matthew Brandon

Maas

Anne Maass

Brian Mac Grory

Michael R. MacAskill

Loch Macdonald

Calixto Machado

Kenneth J. Mack

Ian R. Mackenzie

Colum D. MacKinnon

Tracy E. Madsen

Melinda Magyari

Anja Mähler

Jennifer Juhl

Majersik** $^{* *}$

Andrea Malaspina

Irene A. Malaty

Angela M. Malek

Paresh A. Malhotra

Amer M. Malik

Rainer Malik

Chindo Bala Mallum

Andrew L. Mammen

Sarah Mancone

David E. Mandelbaum

Vishal Mandge

Emmanuel

Mandonnet

Ashutosh K.

Mangalam

Sara Manning

Rebekah Mannix

Tadaaki Mano

Ali Manouchehrinia

Georgios Manousakis

Soe S. Mar

Rohit Marawar

Russell L. Margolis

Romain Marignier

Sandro Marini

Matthew Scott Markert

Aikaterini

Markopoulou

Wilson Marques

Juan Manuel Marquez-

Romero

Connie Marras
Ruth Ann Marrie

Jennifer Marsella

Elisabeth Breese

Marsh

Randolph S.

Marshall**

Daniel Marson

Joan Marti-Fabregas

Wayne Martin

Juan Carlos Martinez

Castrillo

Erika Tatiana

Marulanda-

Londono

Joseph C. Masdeu

Shavonne Massey

Colin L. Masters

Alina Masters-Israilov

Farrah J. Mateen

Susan Matesanz

Gary W. Mathern*

Shaguna Mathur

Makoto Matsui

Emma L. Matthews*

Niklas Mattsson

Alberto Maud*

Alexandre Balzano

Maulaz

Jerome Mawet

Arne May

Elizabeth Rose

Mayeda

Richard Mayeux

Michael Mazya

Anna Teresa Mazzeo

Sara Mazzucco

Justin C. McArthur

Stuart McCarter

Jeffrey C. McClean

Lauren McCollum

Louise D. McCullough

Elizabeth Anne

McCusker

Mollie McDermott

William M. McDonald

Gavin V. McDonnell

Robert McFarland

Brian E. McGeeney

Paul D. McGeoch

Jennifer McGuire

Celia McIntosh

Andrew McKeon

Christopher McLouth

Hugh J. McMillan

Nancy A. McNamara

Kimford J. Meador**

Virginia Meca-Lallana
Adam P. Mecca

William Meehan

Prachi Mehndiratta

Kala M. Mehta

Shyamal H. Mehta

Tapan Mehta

Andreas Georg Otto

Meisel

Wassilios Meissner

Nicte I. Mejia

Susanna Melkas

Giovanni Meola

Steven R. Messe*

Ricka Denise Messer

Roberta Messina

Lambros Messinis

Walter Steven Metzer*

Brett C. Meyer

Michelle M. Mielke

Mitchell Gordon Miglis

Fadi Mikhail

Lukasz Milanowski

Eliza C. Miller

John W. Miller*

Neil R. Miller

Steven P. Miller

Tracey A. Milligan

Mark J. Milstein

Hoon-Ki Paul Min

Mia T. Minen

Jonathan W. Mink

Hassan Mir

Ario Mirian

Nishant K. Mishra

Shubhankar Mishra*

Wendy G. Mitchell

Panayiotis D. Mitsias

Janis Miyasaki

Masashi Mizuguchi

Heidi Moawad**

Jeremy J. Moeller*

Christopher L. Moertel

Siawoosh

Mohammadi

Nimish A. Mohile

J.P. Mohr

Jeremy A. Molad

Jennifer Molano

Joan Montaner

Teshamae S. Monteith

Jacqueline Montes

Majaz Moonis

Karla A. Mora

Rodriguez $^{* *}$

Adeolu Morawo
Francesca Morgante

Joel C. Morgenlander

Lewis B. Morgenstern*

Etsuro Mori

Germán Morís

Elizabeth Mormino

Elena Moro

Andrea Morotti

Nicholas A. Morris

Solomon L. Moshe

Ghassan Moubarak

Tahseen Mozaffar*

Susanne

Muehlschlegel

Christoph Mueller

Notger G. Mueller

Susanne Mueller

Nils Muhlert

Keith W. Muir

Ryan Thomas Muir**

Marco Mula

Sarah B. Mulkey

Peter Müller-Barna

Kirsten R. Muller-Vahl

Heidi Munger Clary

David G. Munoz**

Francesco Muntoni

Paolo A. Muraro

Daniel L. Murman

Santosh B. Murthy

Erik S. Musiek

Krishna Mylavarapu

John Mytinger

Veeresh Kumar N.

Shivamurthy

Duk L. Na

Rima Nabbout

Benedetta Nacmias*

Zurab Nadareishvili

Elie Naddaf

Stephen E. Nadeau

Ahsan Moosa Naduvil

Valappil

Nandakumar

Nagaraja* $^{*}$

Fadi B. Nahab

Stephanie J. Nahas

Kavita V. Nair

Robert T. Naismith

Pushpa

Narayanaswami*

Fábio Augusto

Nascimento**

Avindra Nath

Daniel A. Nation

Anil Neelakantan

Ahmad Nehme*
Maromi Nei

Erin Neil

Kevin R. Nelson

Hermann Neugebauer

Christoph M.

Neuwirth

Joel Neves Briard

Douglas E. Ney*

Yu-tze Ng

Joseph Ngeh

Jennifer M. Nicholas

Fenwick T. Nichols*

Katharine Ames

Nicholson

Katherine Nickels

Anna Nilsson

Melissa J. Nirenberg

Ichizo Nishino

Flavio Nobili

James McCallum

Noble

Michael J. Noetzel

Christian H. Nolte

Gina Norato

Rodrigo Noseda

Bardia Nourbakhsh

John G. Nutt

Marc R. Nuwer

Paul A. Nyquist

Cumara O'Carroll

Yazmin Odia

Steve O’Donnell

Cormac A.

O'Donovan

Christopher S. Ogilvy

Hwamee Oh

Susanna S. O’Kula

Fabricio Ferreira de

Oliveira

Jamary Oliveira-Filho

David John Oliver

Jean Marc Olivot

Heather E. Olson

Osamu Onodera

Marco Onofrj*

Daniel Ontaneda

Kristine O'Phelan

Serena L. Orr

Richard Orrell

Francesco Orzi

Benjamin Osborne

Maryam Oskoui

Adam P. Ostendorf

Padraig O'Suilleabhain

Michael O'Sullivan

Brian R. Ott

Walid Oulehri 
Thure Filskov

Overvad

James Owens

Gennaro Pagano

Francesco Pagnini

Kyoungjune Pak

Raffaele Palladino

Jose-Alberto Palma

Andre Palmini

Sebastian Palmqvist

Jie Pan

Eleni Panagiotakaki

Massimo Pandolfo

Peter K. Panegyres

V. Shane Pankratz**

Leonardo Pantoni**

Francesco Panza

Vasileios Papavasileiou

Gabriel Pardo

Heath R. Pardoe

Davide Pareyson

Maksim Parfyonov

Neal S. Parikh

Yong-Moon Park

Lucilla Parnetti*

Mark W. Parsons

Julio Pascual

Matthew Paul Pase*

Pau Pastor

Anup D. Patel

Manisha Patel

Sheel Pathak

Rachit Patil

Friedemann Paul

Phillip L. Pearl

Otto Pedraza

Victoria Susan Pelak

Sarah T. Pendlebury

Patricia E. Penovich

Umberto Pensato

Renaud Péquignot

Joanna Pera

Joanna E. Perdomo

David L. Perez

Joel S. Perlmutter**

Robert Perneczky

David C. Perry

James R. Perry

M. Scott Perry

Piero Perucca

Elia M. Pestana Knight

B. Lee Peterlin

Nils Peters*

Ruth Peters

Asa Petersen

Corinne Pettigrew

Thanh G. Phan
Fenna Phibbs

Michael S. Phipps

Vincent Picher-Martel

John D. Pickard

William O. Pickrell

Fredrik Piehl

Beatrice S. Pignolet

Yolande A.L.

Pijnenburg

Jagan A. Pillai

Anna L.R. Pinto

Marcus Vinicius R.

Pinto*

Amanda Piquet

Raffaella Pizzolato

Umeton

Peggy J. Planetta*

Joseph J. Pleen

Emily Plowman

Werner Poewe

Anna Poggesi

Michael Polydefkis

Roser Pons

Octavio Marques

Pontes-Neto

Gregory M. Pontone

Aurel Popa-Wagner

Emilio Portaccio

Alyx B. Porter

Brenda E. Porter

Jose Posas

Kathleen L. Poston*

Ron Postuma

Cyril Pottier

Michael H. Pourfar

William J. Powers

Aparna Prabhu

Kameshwar Prasad

Sashank Prasad

Jana Preiningerova

Peter S. Pressman

David C. Preston*

Paolo Preziosa*

Catherine Elizabeth

Crenshaw Price

Michael Privitera

Calin I. Prodan

Amy A. Pruitt

Elizabeth Pulcine

Jukka Putaala

Nataliya Pyatka

Chengxuan Qiu

Carlo Cosimo

Quattrocchi

Aldo Quattrone

Susana Quijano-Roy

Terence J. Quinn*
Alejandro A.

Rabinstein*

Alex D. Rae-Grant*

Mubeen F. Rafay

Jidhin Raj*

Shruti Raja

Yusuf A. Rajabally

Kumar Rajamani

Alexander H. Rajput

Vijay K. Ramanan*

Joel Ramirez

José M. Ramírez-

Moreno

Vesper Fe Marie

Llaneza Ramos

Pedro Ramos-Cabrer

Ciro Ramos-

Estebanez

Stefan Rampp

Anna Ranta

Rashmi Rao

Jaivir S. Rathore

Marcia Hillary Ratner

Radoslav I. Raychev*

Melissa L. Rayhill

Haatem Reda

Jens Peter Reese

Robert William

Regenhardt

Hansotto Reiber

Daniel S. Reich

Stephen G. Reich

Robert I. Reid

Mary M. Reilly

Markus Reindl

Matthias Reinhard

Malin Reinholdsson

Jared P. Reis

Norman R. Relkin

Christel Renoux

William Renthal

Pavle Repovic

Lucas Restrepo

Markus Reuber

Kathryn M. Rexrode

Chandra A. Reynolds

Michael Rezak*

Michael Reznik

Sylvain Rheims

Anette Riisgaard Ribe

Lucia Ricciardi

Gillian I. Rice

Steffi G. Riedel-Heller

Nina Y. Riggins

Jack E. Riggs

Tyler Hyungtaek Rim

E. Bernd Ringelstein
John M. Ringman*

John Robert Rinker

Shannon L. Risacher

Pamela M. Rist

Giovanni Ristori

Eva Katharina Ritzl

Lucia Rivera-Lara

Matthew Stuart

Robbins

Jennifer Robblee*

Angela Roberts

Jodie I. Roberts**

Maisha T. Robinson

Jessica Robinson-Papp

Maria A. Rocca**

Raquel Rocha*

Robert L. Rodnitzky

Amadeo R. Rodriguez

Gustavo J. Rodriguez

Miriam Jocelyn

Rodriguez

Roberto Rodríguez

Rivas*

Leonardo Roever

David J. Roh

Julio C. Rojas

John D. Rolston

Gustavo C. Roman

Jose G. Romano

Javier M. Romero

Carlos G. Romo

Gabriel M. Ronen*

Stefan Ropele

Gary A. Rosenberg

Nicole Rosendale

David B. Rosenfield

Liana Rosenthal

Lindsay A. Ross

Andrea O. Rossetti

Simone Rossi

Elliot J. Roth

Aaron Rothstein**

Dalia Rotstein

Tracey Rouault

Eliane Roulet-Perez

Stasia Rouse

Alex Rovira

Andrew S. Rowland

Bhaskar Roy

Emile P. Roy

Mark N. Rubin*

Anthony Rudd

Steven Rudolph

Stephan J. Rüegg*

Alessandra Rufa

Robert L. Ruff

Ynte M. Ruigrok
Jeffrey Russ**

James A. Russell ${ }^{*}$

Paul Rutecki

Mario Sabatelli

Behnam Sabayan**

Ali S. Saber Tehrani

Severine Sabia

Simona Sacco

Saud A. Sadiq

Reza Sadjadi

Joseph E. Safdieh

Ali A. Saherwala

Monica Saini

Vasu Saini

Helen-Helene SaintHilaire

Ryuta Saito

Albert Saiz

Kalyan Sajja

S. Ahmad Sajjadi

Mohammad Kian

Salajegheh*

Rachel Marie E. Salas*

Michael Martin Saling

Stephen P. Salloway

Amber Salter

Maria Sam

Edgar A. Samaniego

Rohini D.

Samudralwar

Christa O’Hana V. San

Luis

Raquel Sánchez-Valle

Josemir Sander

Stefano Sandrone

Paola Sandroni

Marie Søfteland

Sandvei

Alexander J. Sandweiss

Valeria A. Sansone

David S. Saperstein*

Muralidharan

Sargurupremraj

Rani Sarkis

Amrou Sarraj

Harini Sarva

Marek J. Sasiadek*

Jaume Sastre-Garriga

Douglas Kazutoshi

Sato

Neda Sattarnezhad*

Rodolfo Savica**

Tyson R. Sawchuk

Deanna R. Saylor

Nikolaos Scarmeas

Stephen N. Scelsa*

Catherine Schaefer 
Christof Schaefer

Heidi M. Schambra

Christoph Schankin

Ulrike Schara

Eugene Scharf

Douglas W. Scharre

Ingrid E. Scheffer

Peter D. Schellinger

Carlos H. Schenck

Mark S. Scher

David Schiff

Jeremy D.

Schmahmann

Jens Schmidt

Friedhelm Carl Schmitt

Heike I. Schmolck

Michal Schnaider

Beeri

Michael Joel Schneck

Andrea Lauren

Christman

Schneider

Hauke Schneider

Logan Douglas

Schneider*

Raphael Schneider

Ruth Schneider

Susanne A. Schneider

Michael Schöll

Sonja W. Scholz

Wouter Schonewille

Menno M.

Schoonheim*

Nina F. Schor

Benedikt Schoser

Jonathan M. Schott

John M. Schreiber

Stefanie Schreiber

Stephan U. Schuele

Norbert Schuff

Jörg B.B. Schulz

Courtney Schusse

Stefan Schwab

Jason M. Schwalb

Christopher G.

Schwarz

Heidi Beck Schwarz*

Stefan Schwarz

Neil J. Scolding

Angelo Scuteri

GianPietro Sechi

Lai-Chu See

Ronald Seese

David Julian Seiffge*

James J. Sejvar

Duygu Selcen
John B. Selhorst

Olga Selioutski

Finn Sellebjerg

Johann Sellner

Linda M. Selwa

Souvik Sen

Elizabeth K. Seng

Indranil Sen-Gupta

Sang Won Seo

Esra Serdaroglu*

Jamie Sergeant

Alberto Serrano-Pozo

Laurent Servais

Serenella Servidei

Amir Shaban*

Binit B. Shah

Jharna Nitin Shah

Vishank Arun Shah

Yash D. Shah*

Pashtun Shahim

Kanika Sharma

Louise Sharpe

Basil Sharrack

Jeremy M. Shefner

Kevin Navin Sheth

Michael I. Shevell

Holly Shill

Cheolsu Shin

Robert K. Shin

Shlomo Shinnar

Hitoshi Shinotoh**

Liqi Shu*

Ashfaq Shuaib

Saurabh G. Shukla**

Melissa Shuman

Michael E. Shy

Christos Sidiropoulos

James E. Siegler

Alessio Signori

Stephen D. Silberstein

Brian Silver

Jonathan M. Silver

Julie K. Silver

Faye Silverstein

Gabriella Silvestri

Mauro Silvestrini

Neil G. Simon

Shireen Sindi

Nishant Sinha

Saurabh R. Sinha

Antonio Siniscalchi

Daniel Sirica

Jane Skov

Konstantin Slavin

Eric E. Smith**

Phillip Smith

Todd A. Smitherman
Constance L. SmithHicks

Beth Ellen Snitz

Elson L. So

Riccardo Soffietti

Anja Soldan

Andrew J. Solomon

Claudia Sommer

Sarah Song

Madhu Soni

Maria Pia Sormani

Peter Soros

Andrew $M$.

Southerland

Stephen M. Sozio

Sydney Spagna

Nicole L. Spartano

Marianna Spatola

Roderick Spears

J. David Spence

David C. Spencer

Scott Sperling

Luciano A. Sposato*

Reshmi I. Srinath

Erik K. St. Louis

William Stacey

Nathan P. Staff

John S. Stahl

Michael P.H. Stanley

Jeffrey M. Statland

Robert Staudinger

Hermann Stefan

Maria Stefanidou

Petra Steinacker

Alexis Steinberg

Bernhard J. Steinhoff

Egon Stenager

Brian D. Stephens

Barney J. Stern

Yaakov Stern

Walter F. Stewart

Martin N. Stienen

Jon Stone

Timo Strandberg

Sandra Strazzer

Daniel Strbian

Pasquale Striano

Roy E. Strowd**

Michael Strupp*

Adam Strzelczyk

Olaf Stuve

Jose I. Suarez

Krystal Sully

Charlotte J. Sumner

James F. Sumowski

Shengzhi Darren Sun
Katharina Stibrant

Sunnerhagen

Nicole B. Sur

Rainer Surges

Naoki Suzuki

Anders Svenningsson

Jerry W. Swanson

Walter Swardfager

Michael Swash*

Jeffrey Alan Switzer

Christina Szperka

Minoru Tagawa

Atsushi Takeda

Arturo Tamayo*

Can Ozan Tan*

Eng-King Tan*

Mark Tarnopolsky

Christopher Tarolli

Laura Tassi

Yohei Tateishi

William O. Tatum

Rabi Tawil

Bruce V. Taylor

Frederick R. Taylor

Lynne P. Taylor*

Stefan J. Teipel

Jeanne S. Teitelbaum

Helio A.G. Teive

Jose Francisco Tellez-

Zenteno

Silvia N. Tenembaum

G.M. Terwindt

Charlotte E.

Teunissen

Evan L. Thacker

Vijay M. Thadani

Roshni Thakkar*

Smathorn

Thakolwiboon*

Kiran Teresa Thakur

Mathula Thangarajh

Simon Thebault

William H. Theodore

Dimitrios Theofanidis

Ruth Thiex

Vincent N. Thijs

Liu Lin Thio

Götz Thomalla

George P. Thomas*

P.B.M. Thomas

Robert Joseph

Thomas

Claudius Thome

David M. Thompson

Majda M. Thurnher*

Gretchen E. Tietjen
Ann Henderson

Tilton

Dagmar Timmann

Vincent Timmerman

Philip W. Tipton

Maarten J. Titulaer

Oliver Tobin

Benjamin David

Tolchin*

Jon B. Toledo

Anna-Maija

Tolppanen

Torbjörn Tomson

Silvina Beatriz

Tonarelli

David C. Tong

Shuichi Tonomura

Ahmed T. Toosy

Haluk Topaloglu

James C. Torner

Antonio Toscano

Emmanuel Touzé

Ryan Townley

Kazunori Toyoda

Christopher B.

Traner*

Claudia Trenkwalder

Edwin Trevathan

Ludovic Trinquart

Jaya Trivedi

Maria Trojano

Alexander I. Tröster

Jack W. Tsao*

Benjamin Tseng

Georgios Tsivgoulis

Shoji Tsuji

Anil Man Tuladhar

Yu-Chi Tung

Carmen Tur

Chris Turner

Dana P. Turner

Raymond Scott

Turner

Antonino A.

Tuttolomondo

Kenneth L. Tyler*

William R. Tyor

Ken Uchino

Lucina Q. Uddin

Chinedu T. Udeh-

Momoh

Toshitaka Umemura

Marcus M. Unger

Timo Uphaus

Horst Urbach

Maarten

Uyttenboogaart* 


\begin{tabular}{|c|c|c|c|c|}
\hline Andrei A. Vakhtin & Marcel Verbeek & Yongjun Wang & Auriel Willette & E. Ann Yeh* \\
\hline Peter Valkovic & Steven Vernino* & Braxton B. & Joshua Zebadiah & Crystal J.J. Yeo \\
\hline Philip Van Damme & Paul M. Vespa & Wannamaker & Willey & Jennifer S. Yokoyama \\
\hline Anneke Van der Walt & Angeliki Vgontzas & Alex Ward & Hugh J. Willison & Keir X.X. Yong \\
\hline J. Gert Van Dijk & Marie Vidailhet & Seth A. Warschausky & Duncan Wilson & Marcy Yonker \\
\hline Pieter A. van Doorn & Thomas Vidic & Katja E. Wartenberg* & Robert S. Wilson & Sohei Yoshimura \\
\hline Thilo Van Eimeren & Matti H. Viitanen & Patrick J. Waters & Jeffrey J. Wing & Mitsuhiro Yoshita \\
\hline Baziel G. van Engelen & George K. Vilanilam & John A. Waterston & Y.K. Wing & G. Bryan Young \\
\hline Willemijn van Erp & Vicente Villanueva & Reem Waziry & Dean M. Wingerchuk & Kate C. Young \\
\hline Ellis S. van Etten & Pablo Villoslada & Alastair J.S. Webb & Thomas Wingo & Robert J. Young \\
\hline Jay A. van Gerpen* & Francois J.G. & Corinna Weber- & Elaine C. Wirrell & William B. Young \\
\hline Jamie Joseph Van & Vingerhoets* & Schoendorfer & Jens Witsch** & Shengyuan $\mathrm{Yu}$ \\
\hline Gompel & Mellekate S. Vishwas* & Lawrence R. Wechsler & Karsten Witt & Derek Yuan \\
\hline Keith P. Van Haren & Jerry Vockley & Jill Wecht & Max Wiznitzer & Nathan Zasler \\
\hline Koen J. Van Laere & Paula Emanuela & Jesse M. Weinberger & Yohannes Woubishet & Alonso Gonzalo Zea \\
\hline Klaus Van Leyen & Voinescu & Brian G. Weinshenker & W. Woldeamanuel & Vera $^{* *}$ \\
\hline Paul C. Van Ness & Sarah Vollbracht & Sandra Weintraub & Nicole I. Wolf & Anastasia Zekeridou \\
\hline Robert J. van & Nirali A. Vora & Sven Wellmann & Peter Wolf & Johan Zelano \\
\hline Oostenbrugge & Stephanie J.B. Vos & Rebecca Erwin Wells & Stefan Wolf & Aaron Simon \\
\hline Michel J.A.M. van & Rhonda R. Voskuhl & Patrick Y. Wen & Gil I. Wolfe & Zelikovich \\
\hline Putten & Andrew J. Waclawik & John Kent Werner* & Frank J. Wolters & Henrik Zetterberg* \\
\hline Gregory P. Van & Hatem Wafa & David John Werring & Daniel Woo & Kirsten E. Zeuner \\
\hline Stavern & Mark Wainwright & Mary Ann Werz & Elisabeth McCarty & Ting Zhang \\
\hline Susanne J. van Veluw & Tobias Walbert & Patrick Weydt & Wood & Chen Zhao* \\
\hline Nicola Vanacore & Francis O. Walker & Martin Weygandt & John M. Worthington & Henry Zhao \\
\hline Rik Vandenberghe & Harrison Walker & Lawrence J. Whalley* & Marcella Wozniak & Chongke Zhong \\
\hline Wim Vandenberghe & Keenan A. Walker & John R. Wherrett & Ruey-Meei Wu & An Zhou \\
\hline Panayiotis N. Varelas & Ruth H. Walker & Jennifer L. Whitwell & Courtney J. Wusthoff* & Zhengbao Zhu* \\
\hline Alejandro Vargas & Michael Wall & Wolfgang Wick & Michael L. Wynn & Wendy C. Ziai \\
\hline Jose F. Varona & Mitchell T. Wallin & Matthew P. Wicklund & Huichun Xu & Daniel Zielonka \\
\hline Arthur Varoquaux & Miranda Wan & Meredith Wicklund & Junqian Xu & Sasa Zivkovic \\
\hline Andrea Varrone & Britta Wandschneider & Heinz Wiendl & Shadi Yaghi & Jose Rafael Pantoja \\
\hline Hartmut Vatter & Annabel Kim Wang & Gerald J. Wiest & Dixon Yang* & Zuzuarregui \\
\hline Aravindhan & Danny J.J. Wang & Eelco F.M. Wijdicks & Hiroshi Yao & \\
\hline Veerapandiyan** & Irene Wang & Einar P.V. Wilder- & Sevil Yasar & \\
\hline Arun Venkatesan & Tian Wang* & Smith & John Robert Yates & \\
\hline
\end{tabular}

\section{Disclosure}

The authors report no disclosures relevant to the manuscript.

Go to Neurology.org/N for full disclosures. 


\title{
Neurology
}

\author{
Message From the Editors to Our Reviewers \\ José G. Merino, Olga Ciccarelli, Bradford B. Worrall, et al. \\ Neurology 2021;96;1-9 \\ DOI 10.1212/WNL.0000000000011204
}

This information is current as of December 28, 2020

$\begin{array}{ll}\begin{array}{l}\text { Updated Information \& } \\ \text { Services }\end{array} & \begin{array}{l}\text { including high resolution figures, can be found at: } \\ \text { http://n.neurology.org/content/96/1/1.full }\end{array} \\ \text { Permissions \& Licensing } & \begin{array}{l}\text { Information about reproducing this article in parts (figures,tables) or in } \\ \text { its entirety can be found online at: } \\ \text { http://www.neurology.org/about/about_the_journal\#permissions }\end{array} \\ \text { Reprints } & \begin{array}{l}\text { Information about ordering reprints can be found online: } \\ \text { http://n.neurology.org/subscribers/advertise }\end{array}\end{array}$

Neurology $®$ is the official journal of the American Academy of Neurology. Published continuously since 1951, it is now a weekly with 48 issues per year. Copyright @ 2020 American Academy of Neurology. All rights reserved. Print ISSN: 0028-3878. Online ISSN: 1526-632X.

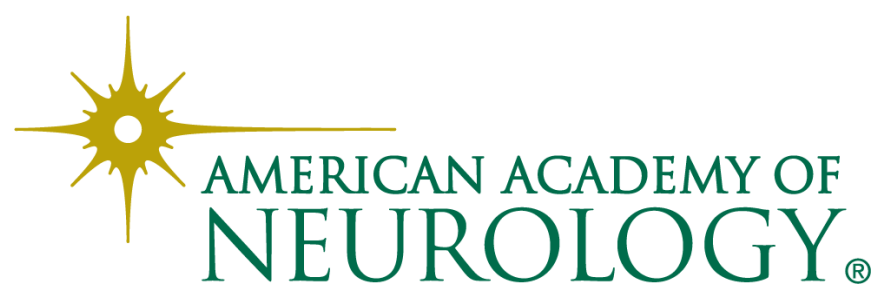

